\title{
新型 5,6,7,8-四氢苯并噻吩并嘧啶酮衍生物的合成与抗肿瘤活性研究
}

\author{
王红梅 $^{a} \quad$ 郭树兵 ${ }^{b}$ 胡扬根*,a,c 曾小华 ${ }^{a}$ 杨光义*,a,c \\ ( ${ }^{a}$ 武当特色中药研究湖北省重点实验室 湖北医药学院医学化学研究所 十堰 442000) \\ ( ${ }^{b}$ 湖北医药学院图书馆 十堰 442000) \\ ( ${ }^{c}$ 湖北医药学院附属太和医院药学部 十堰 442000)
}

\begin{abstract}
摘要 应用 $5,6,7,8$-四氢苯并噻吩膦亚胺(2)与烷基异氰酸酯的氮杂 Wittig 反应生成碳二亚胺 $\mathbf{2}$, 在不同的条件下, $\mathbf{2}$ 分别 与不同的亲核试剂反应, 有效地合成了不同取代的新型稠合噻吩并[2,3- $d$ ] 嘧啶-4(3H)-酮衍生物. 所得化合物的结构由 ${ }^{1} \mathrm{H}$ NMR, IR, MS 和元素分析所确证. 初步的体外抗肿瘤活性测试, 结果显示目标化合物具有抑制肿瘤细胞的生长, 其 中 $\mathbf{5 d}$ 对口腔肿瘤细胞 $\mathrm{KB} 2 \mathrm{IC}_{50}$ 值为 $19.0 \mu \mathrm{mol} / \mathrm{L}$ ，表现出潜在的抗肿瘤活性.
\end{abstract}

关键词 氮杂Wittig 反应; 噻吩并嘧啶酮; 合成; 抗肿瘤活性

\section{Synthesis and Antitumor Activity of Some Novel 5,6,7,8-Tetrahydro- benzo-thieno[2,3-d]pyrimidin-4(3H)-one Derivatives}

\author{
Wang, Hongmei $^{a} \quad$ Guo, Shubing ${ }^{b} \quad$ Hu, Yanggen ${ }^{*, a, c} \quad$ Zeng, Xiaohua ${ }^{a} \quad$ Yang, Guangyi*a,c \\ ( ${ }^{a}$ Hubei Key Laboratory of Wudang Local Chinese Medicine Research and Institute of Medicinal Chemistry, \\ Hubei University of Medicine, Shiyan 442000) \\ $\left({ }^{b}\right.$ The Library of Hubei University of Medicine, Shiyan 442000) \\ ( ${ }^{c}$ Department of Pharmacy, Taihe Hospital of Hubei University of Medicine, Shiyan 442000)
}

\begin{abstract}
The aza-Wittig reaction of iminophosphorane $\mathbf{2}$ with alkyl isocyanates gave carbodiimides $\mathbf{3}$, which were allowed to react with various nucleophilic reagents under mild condition in satisfactory yields to prepare a novel series of 5,6,7,8-tetrahydrobenzothieno[2,3- $d]$ pyrimidin-4(3H)-one derivatives. The structures of these compounds were confirmed by ${ }^{1} \mathrm{H}$ NMR, IR, MS and elemental analysis. The in vitro antitumor activities of compounds were analyzed with 3-(4,5-dimethylthiazol-2-yl)-2,5-diphenyltetrazo-liumbromide (MTT) standard method, 5d stood out as the most potent showing an $\mathrm{IC}_{50}$ of $19.0 \mu \mathrm{mol} / \mathrm{L}$ against human tumor cell lines $(\mathrm{KB})$.
\end{abstract}

Keywords aza-Wittig reaction; thieno[2,3- $d$ ]pyrimidin-4(3H)-ones; synthesis; antitumor

噻吩并嘧啶衍生物是一类具有良好的生物活性和 药理活性的杂环化合物, 早在 20 世纪 70 年代就备受人 们的注意. 许多噻吩并嘧啶酮的衍生物具有杀菌、抗滤 过性病原体、抗惊厥及除草等活性, 有些作为镇定剂 ${ }^{[1,2]}$. 如 2-烷氧基或 2-烷基取代的噻吩并嘧啶酮衍生物具有 良好的杀菌和抗菌活性 ${ }^{[3]}$. 最近研究表明一些噻酚并嘧 啶衍生物通过抑制不同的蛋白激酶表现出潜在的抗肿 瘤活性(图 1) ${ }^{[4 \sim 6]}$, 如吗啡啉取代的噻吩并嘧啶(GDC-
0941) 是一种有效的 PI3K 激酶抑制剂; 化合物 I 和 II 是 潜在的 EGFR 抑制剂, 对多种肿瘤细胞的生长都表现出 一定的抑制作用.

氮杂 Wittig 反应因其具有反应原料易得、反应条件 温和、反应选择性好等特点, 已成为一种合成氮杂环的 有效手段 ${ }^{[7 ~ 11]}$. 我们课题组已报道应用 aza-Wittig 反应, 以简易的操作方法，有效地合成了 3 -芳基取代的 $5,6,7,8$-四氢苯并噻吩 $[2,3-d]$ 并嘧啶酮衍生物 ${ }^{[12]}$. 在此基

\footnotetext{
*E-mail: huyg@hbmu.edu.cn, ygy999@163.com.

Received October 24, 2014; revised December 15, 2015; published online January 5, 2015

Project supported by the Natural Science Foundation of Hubei Province (No. 2011CDC006), the Fund of Hubei 2011 Cooperative Innovation Center (No. 2011JH-2014CXTT07) and the Science and Technology Innovation team Project of Hubei University of Medicine (Nos. 2011CXX03, 2009QDJ15, 2014XKJSXJ06).

湖北自然科学基金(No. 2011CDC006)、湖北省 2011 协同创新中心基金(No. 2011JH-2014CXTT07)和湖北医药学院优秀中青年科技创新团队研究(Nos. 2011CXX03, 2009QDJ15, 2014XKJSXJ06)资助项目.
} 


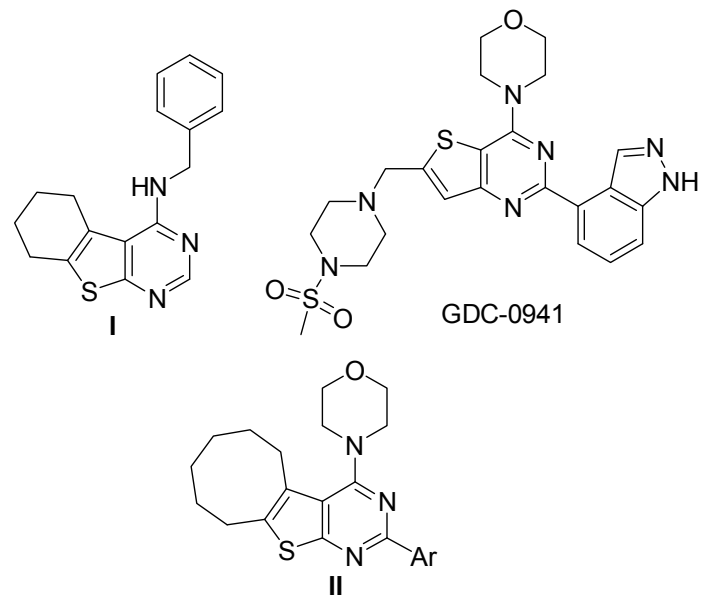

图 1 具有潜在抗肿瘤活性的噻吩并嘧啶衍生物

Figure 1 A number of thienopyrimidinones were reported as anticancer agents

础上, 我们进一步探讨 aza-Wittig 反应的应用, 以简易 的操作方法, 有效地合成新型的 3-烷基取代的 5,6,7,8四氢苯并噻吩 $[2,3-d]$ 并嘧啶酮衍生物, 初步篮选了其抗 肿瘤活性.

参考文献[13]方法, 合成中间体膦亚胺 2 . 合成路线 见 Scheme 1.

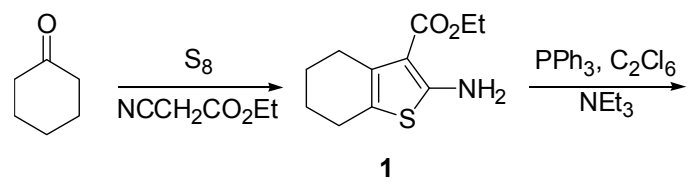<smiles>C=NPC=Nc1sc2c(c1C(=O)OCC)CCCC2</smiles>

图式 1 膦亚胺 2 的合成

Scheme 1 Preparation of iminophosphorane 2

膦亚胺 $\mathbf{2}$ 与烷基异氧酸酯反应, 得到的碳二亚胺 $\mathbf{3}$ 与仲胺反应, 生成类胍中间体 $\mathbf{4 , 4}$ 经醇钠催化关环, 即 得到 2-二烷氨基-5,6,7,8-四氢苯并噻吩并嘧啶酮(5). 3 与 醇及相应醇钠反应，直接得到 2 -烷氧基-5,6,7,8-四氢苯 并噻吩 $[2,3-d]$ 并嘧啶酮 (6); 3 与酚在无水碳酸钾催化下 反应，制备了 2-芳氧基-5,6,7,8-四氢苯并噻吩 $[2,3-d]$ 并 嘧啶酮(7). 该方法原料易得、条件温和、收率较高. 合 成路线见 Scheme 2, 化合物见表 1 .

\section{1 结果与讨论}

\section{1 反应条件}

参考文献[14]方法, 在乙醇溶液中, 等物质的量的

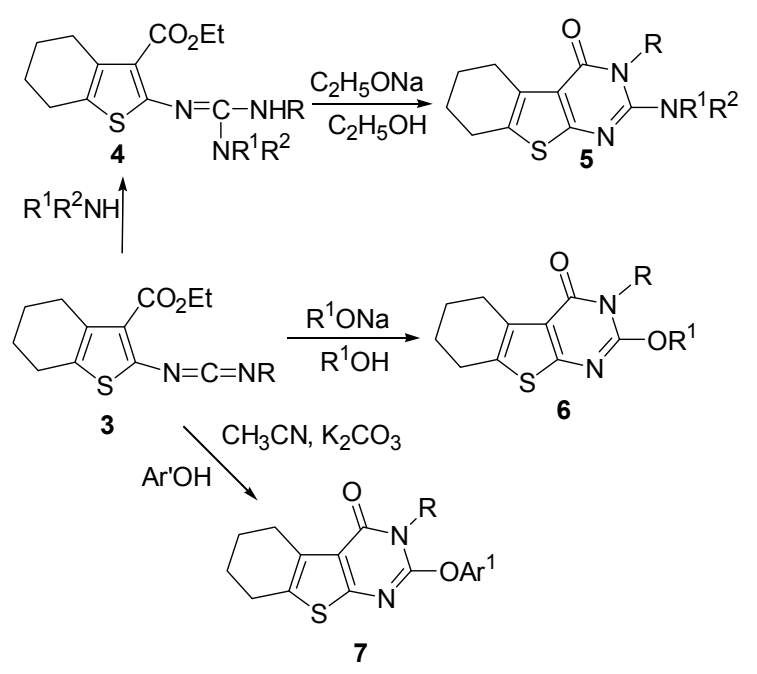

图式 $25,6,7,8$-四氢苯并噻吩并嘧啶酮衍生物 $5 、 6$ 和 7 的合 成

Scheme 2 Preparation of 5,6,7,8-tetrahydrobenzothieno[2,3- $d]$ pyrimidin-4(3H)-one 5, $\mathbf{6}$ and $\mathbf{7}$

表 $15,6,7,8$-四氢苯并噻吩并嘧啶酮衍生物 $5 、 6$ 和 7 的制备 Table 1 Preparation of 5,6,7,8-tetrahydrobenzothieno[2,3- $d$ ]pyrimidin-4(3H)-one 5, 6 and 7

\begin{tabular}{|c|c|c|}
\hline Compd. & $\mathrm{R}$ & $\mathrm{R}^{1} \mathrm{R}^{2}\left(\mathrm{R}^{1}\right.$ or $\left.\mathrm{Ar}^{1}\right)$ \\
\hline $5 \mathbf{a}$ & $\mathrm{CH}_{2} \mathrm{CH}_{2} \mathrm{CH}_{2} \mathrm{CH}_{3}$ & \\
\hline $5 b$ & $\mathrm{CH}_{2} \mathrm{CH}_{2} \mathrm{CH}_{2} \mathrm{CH}_{3}$ & $\mathrm{CH}\left(\mathrm{CH}_{3}\right)_{2}$ \\
\hline $5 c$ & $-\mathrm{CH}_{2}$ & $\mathrm{CH}\left(\mathrm{CH}_{3}\right)_{2}$ \\
\hline $5 \mathbf{d}^{a}$ & $-\mathrm{CH}_{2}$ & $\mathrm{CH}_{2} \mathrm{CH}_{2} \mathrm{CH}_{2} \mathrm{CH}_{3}$ \\
\hline $6 \mathbf{a}$ & $\mathrm{CH}\left(\mathrm{CH}_{3}\right)_{2}$ & $\mathrm{CH}_{2} \mathrm{CH}_{3}$ \\
\hline $\mathbf{6 b}^{a}$ & $\mathrm{CH}\left(\mathrm{CH}_{3}\right)_{2}$ & $\mathrm{CH}_{2} \mathrm{CH}_{2} \mathrm{CH}_{3}$ \\
\hline $6 c$ & $\mathrm{CH}\left(\mathrm{CH}_{3}\right)_{2}$ & $\mathrm{CH}_{2} \mathrm{CH}_{2} \mathrm{CH}_{2} \mathrm{CH}_{3}$ \\
\hline 6d & $\mathrm{CH}_{2} \mathrm{CH}_{2} \mathrm{CH}_{2} \mathrm{CH}_{3}$ & $\mathrm{CH}_{2} \mathrm{CH}_{3}$ \\
\hline $6 e$ & $\mathrm{CH}_{2} \mathrm{CH}_{2} \mathrm{CH}_{2} \mathrm{CH}_{3}$ & $\mathrm{CH}_{2} \mathrm{CH}_{2} \mathrm{CH}_{3}$ \\
\hline $6 f$ & $\mathrm{CH}_{2} \mathrm{CH}_{2} \mathrm{CH}_{2} \mathrm{CH}_{3}$ & $\mathrm{CH}_{2} \mathrm{CH}_{2} \mathrm{CH}_{2} \mathrm{CH}_{3}$ \\
\hline $7 \mathbf{a}$ & $\mathrm{CH}\left(\mathrm{CH}_{3}\right)_{2}$ & \\
\hline $7 b$ & $\mathrm{CH}\left(\mathrm{CH}_{3}\right)_{2}$ & $\mathrm{H}_{3} \mathrm{C}^{-}$ \\
\hline $7 \mathrm{c}$ & $\mathrm{CH}_{2} \mathrm{CH}_{2} \mathrm{CH}_{2} \mathrm{CH}_{3}$ & $\mathrm{H}_{3} \mathrm{C}$ \\
\hline
\end{tabular}


续表

\begin{tabular}{lll}
\hline Compd. & $\mathrm{R}$ & $\mathrm{R}^{1} \mathrm{R}^{2}\left(\mathrm{R}^{1}\right.$ or $\left.\mathrm{Ar}^{1}\right)$ \\
\hline $7 \mathbf{d}$ & $\mathrm{CH}_{2} \mathrm{CH}_{2} \mathrm{CH}_{2} \mathrm{CH}_{3}$ & \\
$7 \mathbf{7 f}$ & $\mathrm{CH}_{2} \mathrm{CH}_{2} \mathrm{CH}_{2} \mathrm{CH}_{3}$ & \\
& $\mathrm{CH}_{2} \mathrm{CH}_{2} \mathrm{CH}_{2} \mathrm{CH}_{3}$ &
\end{tabular}

${ }^{a}$ 已知化合物 ${ }^{[13]}$.

氰乙酸乙酯和环己酮在二乙胺催化下, 制备 2-氨基4,5,6,7-四氢苯并噻吩-3-甲酸乙酯(1), 1 进一步和三苯基 膦和六氯乙烷、三乙胺反应制备了膦亚胺 2 .

在 $40 \sim 50{ }^{\circ} \mathrm{C}$ 条件, 用干燥氮气保护, 膦亚胺 2 和 烷基异氭酸酯反应生成碳二亚胺 $\mathbf{3}$. 碳二亚胺 $\mathbf{3}$ 与胺反 应，生成胍中间体 4, 4 在乙醇钠作用下得到化合物 5; 碳二亚胺 3 与酚反应, 在碳酸钾催化下, 直接关环生成 化合物 6; 碳二亚胺 3 直接与醇及相应醇钠反应得到化 合物 7. 值得关注的是碳二亚胺 3 与伯胺(如芐胺)的反 应, 理论上应该有两种产物 5d 和 5d' (Scheme 3), 但是 采用仲胺相同方法, 仅得到一种关环产物. 5d 的形成, 根据胍中间体在乙醇钠催化作用下，取代基空间位阻和 氨基亲核性的作用, 自发地发生关环反应, 结果选择性 是唯一的.

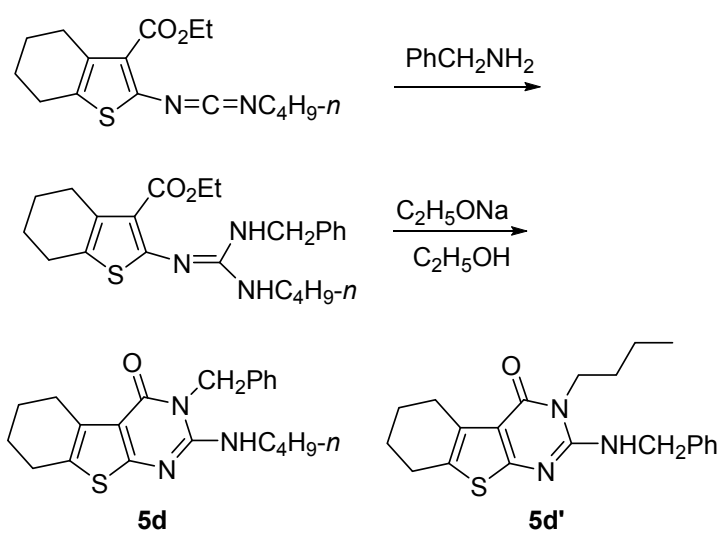

图式 $35,6,7,8$-四氢苯并噻吩并嘧啶酮(5d)的合成 Scheme 3 Preparation of 5,6,7,8-tetrahydrobenzothieno[2,3- $d$ ]pyrimidin-4(3H)-one (5d)

\section{2 目标化合物 5、6 和 7 的波谱特征}

目标化合物 5、6 和 7 的结构经过 IR、 ${ }^{1} \mathrm{H}$ NMR, EI-MS 和元素分析确认. 在该系列化合物的 ${ }^{1} \mathrm{H}$ NMR 中, 化合物 5、6 和 7 中 5,6,7,8-碳环上氢的化学位移在 $\delta$ 1.60 2.98 之间呈现出多重峰. 例如化合物 $5 \mathbf{d}$ 的 ${ }^{1} \mathrm{H}$
NMR 中苄基亚甲基的化学位移在 $\delta 5.25$ 显示单峰, 丁 胺基的 $\mathrm{NH}$ 和 $\mathrm{NCH}_{2}$ 分别在 $\delta 4.37,3.31$ 处显示一单峰和 三重峰, 其它各氢原子的化学位移都与 ${ }^{1} \mathrm{H} N \mathrm{NR}$ 谱图中 一一对应. 在 IR 谱图中, 目标化合物 5、6 和 7 嘧啶酮 $\mathrm{C}=\mathrm{O}$ 的强伸缩振动特征吸收峰都处在 $1685 \mathrm{~cm}^{-1}$ 左右. 在 MS 谱图中, 目标化合物都有较强的分子离子峰，主 要碎片离子峰都与预期的目标分子结构相吻合. 此外为 了能够更清楚地确认此类化合物的结构, 前期我们对化 合物 5d 和 $6 \mathbf{b}$ 等都有相应的晶体结构研究 ${ }^{[13]}$.

\section{3 化合物 4 和 5 的抗肿瘤活性}

\subsection{1 细胞培养}

口腔癌和肝癌、卵巢癌细胞株( $\mathrm{KB}$ and HepG2, OVCAR3)用 RPMI-1640 培养液于 $\mathrm{CO}_{2}$ 孵箱中 $37{ }^{\circ} \mathrm{C}$ 、 $5 \% \mathrm{CO}_{2}$ 饱和湿度下培养. 贴壁细胞用 $0.25 \%$ 胰蛋白酶 消化传代.

\subsubsection{MTT 比色法}

将处于对数生长期的细胞制成单细胞悬液，调整细 胞浓度为 $5 \times 10^{4}$ 个 $/ \mathrm{mL}$ ，接种于 96 孔培养板，每孔接种 $100 \mu \mathrm{L}$, 每孔加入不同浓度的药物 $100 \mu \mathrm{L}$ 受试药, 使初 步篎选的药物终浓度分别为 $5.0 、 20.0 、 50.0 、 100 \mu \mathrm{mol} / \mathrm{L}$, 另设阴性对照组(不加药)和阳性对照(顺铂), 每组均设 4 个复孔, 培养 $24 \mathrm{~h}$, 吸弃上清后加噻唑蓝(MTT) (5 $\mathrm{mg} / \mathrm{mL}$ ) $100 \mu \mathrm{L}$, 弃去上清液，加二甲基亚砜(DMSO) $100 \mu \mathrm{L}$, 在平板摇床上摇匀, 用全自动酶联免疫检测仪 于 $495 \mathrm{~nm}$ 波长处测定吸光度 $(\mathrm{OD})$ 值, 求出 $\mathrm{IC}_{50}$. 整个试 验操作都在湖北医药学院第三附属医院药学研究所完 成.

抑制率 $/ \%=\left[A_{495}\right.$ (阴性对照) $-A_{495}$ (加药组) $) /$ $A_{495}$ (阴性对照) $\times 100 \%$

\subsection{3 结果}

实验数据以 $\chi \pm s$ 表示, 数据分析采用 SPSS10.0 统 计软件进行. 用 Origin 软件, 通过半对数拟合直线求 $\mathrm{IC}_{50}$ 值. 结果显示化合物 4 和 $\mathbf{5}$ 对不同的肿瘤细胞口腔 癌和肝癌、卵巢癌细胞株(KB 和 HepG2, OVCAR3)具有 一定的抑制作用(表 2).

\section{2 结论}

我们应用 aza-Wittig 反应，在温和的条件下，采用 易得的原料，以简单的操作方法，有效地合成新型的多 取代的 5,6,7,8-四氢苯并噻吩 [2,3-d]并嘧啶酮衍生物. 通 过体外药物活性试验, 测定了化合物分别对口腔癌和肝 癌、卵巢癌细胞株(KB and HepG2, OVCAR3)生长的抑 制活性，结果显示该类化合物具有潜在的抗肿瘤活性， 如化合物 $\mathbf{5 d}$ 对口腔癌细细胞 $\mathrm{KB}$ 的 $\mathrm{IC}_{50}$ 值为 19.1 
表 $25,6,7,8$-四氢苯并噻吩并嘧啶酮衍生物 $5 、 6$ 和 7 的抗肿 瘤活性 ${ }^{a}$

Table 2 Antitumor activities of 5,6,7,8-tetrahydrobenzothieno$[2,3-d]$ pyrimidin-4(3H)-ones $\mathbf{5 , 6}$ and 7

\begin{tabular}{ccccr}
\hline \multirow{2}{*}{ Entry } & \multirow{2}{*}{ Compd. } & \multicolumn{3}{c}{$\mathrm{IC}_{50}\left(\mu \mathrm{mol} \cdot \mathrm{L}^{-1}\right)$} \\
\cline { 3 - 5 } & $\mathbf{5 a}$ & 89.1 & $>100$ & 96.4 \\
2 & $\mathbf{5 b}$ & 33.7 & 53.1 & 33.3 \\
3 & $\mathbf{5 c}$ & 78.6 & 40.6 & 59.8 \\
4 & $\mathbf{5 d}$ & $\mathbf{1 9 . 0}$ & $>100$ & 48.6 \\
5 & $\mathbf{6 a}$ & $30<\mathrm{IC}_{50}<100$ & 40.6 & 47.6 \\
6 & $\mathbf{6 b}$ & $>100$ & 51.5 & 64.1 \\
7 & $\mathbf{6 c}$ & $>100$ & $>100$ & 62.5 \\
8 & $\mathbf{6 d}$ & $>100$ & $>100$ & $>100$ \\
9 & $\mathbf{6 e}$ & $>100$ & $>100$ & $>100$ \\
10 & $\mathbf{6 f}$ & $>100$ & $>100$ & $>100$ \\
11 & $\mathbf{7 a}$ & $\mathbf{2 7 . 1}$ & 99.0 & $>100$ \\
12 & $\mathbf{7 b}$ & 84.5 & $>100$ & $>100$ \\
13 & $\mathbf{7 c}$ & 34.2 & $>100$ & 46.9 \\
14 & $\mathbf{7 d}$ & 77.8 & $>100$ & $>100$ \\
15 & $\mathbf{7 e}$ & 82.2 & $>100$ & $\mathbf{2 2 . 7}$ \\
16 & $\mathbf{7 f}$ & $>100$ & $>100$ & 73.1 \\
对照 & 顺铂 & - & - & 36.3 \\
\hline$a$ “一” 表示未做阳性对照试验. & &
\end{tabular}

$\mu \mathrm{mol} / \mathrm{L}$, 此研究进一步扩展了 aza-Wittig 反应的应用, 同时为抗肿瘤药物的研究奠定了一定的研究基础.

\section{3 实验部分}

\section{1 仪器与试剂}

熔点用北京第三光学仪器厂生产的 X4 型熔点仪测 定, 温度计未经校正; 红外以 Perkin. Elemer PE-983 红 外光谱仪测定: NMR 用 Varian Mercury 400 型 $400 \mathrm{MHz}$ 核磁共振仪测定, TMS 为内标; MS 使用 FinniganTrace 质谱仪测定; 元素分析使用 Vario EL III 元素分析仪测 定. 所用试剂为国产(或进口)化学纯或分析纯.

\section{2 实验方法}

用干燥氮气保护, 膦亚胺 $\mathbf{2}^{[15]}(3 \mathrm{mmol})$ 的二氯甲烷 溶液加入等物质的量的烷基异氧酸酯, 在 $40 \sim 50{ }^{\circ} \mathrm{C}$ 反 应 $8 \sim 10 \mathrm{~h}$, 减压脱去大部分溶剂, 加入 $V$ (乙醚) : $V$ (石 油醚 $)=1: 2(15 \sim 20 \mathrm{~mL})$ 的混合溶液, 过滤以除去三苯 基膦, 滤液在减压条件下脱去溶剂即得碳二亚胺 3.3 无 需进一步纯化, 可直接进行下一步操作. 将所得碳二亚 胺 3 溶于二氯甲烷 (15 mL) 中, 滴加相应的仲胺 (3 $\mathrm{mmo1}$ ), 在室温下反应 $1 \mathrm{~h}$, 所得到的类胍中间体 4 无需 进一步纯化, 可直接进行下一步操作. 脱去所得中间体 4 的溶剂, 加入无水乙醇 $(10 \mathrm{~mL})$ 和几滴乙醇钠的无水乙 醇溶液, 在室温下反应 $10 \mathrm{~h}$, 减压蒸去溶剂, 残余物以 乙醇或二氯甲烷/石油醚重结晶, 得 2-二烷氨基-3-烷基$5,6,7,8$-四氢苯并噻吩 $[2,3-d]$ 并嘧啶酮衍生物 $\mathbf{5 a} \sim \mathbf{5 c}$. 2-吗啉-3-丁基-5,6,7,8-四氢苯并噻吩并嘧啶酮(5a): 白色固体 $(0.7 \mathrm{~g}, 70 \%)$. m.p. $118 \sim 119{ }^{\circ} \mathrm{C} ;{ }^{1} \mathrm{H}$ NMR $\left(\mathrm{CDCl}_{3}, 400 \mathrm{MHz}\right) \delta$ : $4.08\left(\mathrm{t}, J=7.2 \mathrm{~Hz}, 2 \mathrm{H}, \mathrm{NCH}_{2}\right), 3.84$ (t, $\left.J=4.8 \mathrm{~Hz}, 4 \mathrm{H}, 2 \times \mathrm{CH}_{2}\right), 3.14(\mathrm{t}, J=4.8 \mathrm{~Hz}, 4 \mathrm{H}, 2 \times$ $\left.\mathrm{CH}_{2}\right), 3.00 \sim 2.73\left(\mathrm{~m}, 4 \mathrm{H}, 2 \mathrm{CH}_{2}\right), 1.87 \sim 1.70(\mathrm{~m}, 6 \mathrm{H}, 3 \times$ $\left.\mathrm{CH}_{2}\right), 1.38 \sim 1.31\left(\mathrm{~m}, 2 \mathrm{H}, \mathrm{CH}_{2}\right), 0.95(\mathrm{t}, J=7.2 \mathrm{~Hz}, 3 \mathrm{H}$, $\left.\mathrm{CH}_{3}\right) ;{ }^{13} \mathrm{C}$ NMR $\left(\mathrm{CDCl}_{3}, 100 \mathrm{MHz}\right) \delta: 13.8,20.3,22.4$, 23.1, 25.2, 25.6, 30.7, 43.9, 51.2, 66.5, 119.5, 131.3, 131.4, 155.5, 159.9, 161.9; IR (KBr) $v: 1667(\mathrm{C}=\mathrm{O}) \mathrm{cm}^{-1}$; MS $m / z(\%): 347\left(\mathrm{M}^{+}, 100\right), 290$ (23), 260 (16), 55 (14). Anal. calcd for $\mathrm{C}_{18} \mathrm{H}_{25} \mathrm{~N}_{3} \mathrm{O}_{2} \mathrm{~S}$ : C 62.22, H 7.25, N 12.09; found C 62.09, H 7.23, N 12.15.

2-异丙氨基-3-丁基-5,6,7,8-四氢苯并噻吩并嘧啶酮 (5b): 白色固体(0.58 g, 61\%). m.p. 83 84 ${ }^{\circ} \mathrm{C} ;{ }^{1} \mathrm{H}$ NMR $\left(\mathrm{CDCl}_{3}, 400 \mathrm{MHz}\right) \delta: 4.29 \sim 4.26(\mathrm{~m}, 2 \mathrm{H}, \mathrm{NH}$ and $\mathrm{CH})$, $3.93\left(\mathrm{t}, J=7.6 \mathrm{~Hz}, 2 \mathrm{H}, \mathrm{NCH}_{2}\right), 2.94 \sim 2.65(\mathrm{~m}, 4 \mathrm{H}, 2 \times$ $\left.\mathrm{CH}_{2}\right), 1.85 \sim 1.60\left(\mathrm{~m}, 6 \mathrm{H}, 3 \times \mathrm{CH}_{2}\right), 1.48 \sim 1.40(\mathrm{~m}, 2 \mathrm{H}$, $\left.\mathrm{CH}_{2}\right), 0.98\left(\mathrm{t}, J=7.2 \mathrm{~Hz}, 3 \mathrm{H}, \mathrm{CH}_{3}\right) ; \mathrm{IR}(\mathrm{KBr}) v: 3367(\mathrm{~N}-$ $\mathrm{H}), 1652(\mathrm{C}=\mathrm{O}) \mathrm{cm}^{-1}$; $\mathrm{MS} \mathrm{m} / z(\%): 320\left(\mathrm{M}^{+}+1,100\right)$, 302 (14), 264 (28), 248 (22), 43 (87). Anal. calcd for $\mathrm{C}_{17} \mathrm{H}_{25} \mathrm{~N}_{3}$ OS: C 63.91, H 7.89, N 13.15; found C 63.71, H 7.93, N 13.34.

2-异丙氨基-3-芐基-5,6,7,8-四氢苯并噻吩并嘧啶酮 (5c): 白色固体(0.76 g, 72\%). m.p. 101 102 ${ }^{\circ} \mathrm{C} ;{ }^{1} \mathrm{H}$ NMR $\left(\mathrm{CDCl}_{3}, 400 \mathrm{MHz}\right) \delta: 7.37 \sim 7.23(\mathrm{~m}, 5 \mathrm{H}, \mathrm{Ar}-\mathrm{H})$, $5.24\left(\mathrm{~s}, 2 \mathrm{H}, \mathrm{NCH}_{2}\right), 4.21(\mathrm{~d}, J=6.8 \mathrm{~Hz}, 1 \mathrm{H}, \mathrm{NH}), 4.12 \sim$ $4.07(\mathrm{~m}, 1 \mathrm{H}, \mathrm{NCH}), 3.00 \sim 2.67\left(\mathrm{~m}, 4 \mathrm{H}, 2 \times \mathrm{CH}_{2}\right), 1.87 \sim$ $1.82\left(\mathrm{~m}, 4 \mathrm{H}, 2 \times \mathrm{CH}_{2}\right), 1.02\left(\mathrm{~d}, J=6.4 \mathrm{~Hz}, 6 \mathrm{H}, 2 \times \mathrm{CH}_{3}\right)$; ${ }^{13} \mathrm{C} \mathrm{NMR}\left(\mathrm{CDCl}_{3}, 100 \mathrm{MHz}\right) \delta$ : 22.5, 22.6,23.3, 25.0, 25.7, 43.7, 43.8, 114.2, 126.6, 127.0, 128.2, 129.3, 131.1, 135.4, 150.1, 159.0, 165.2; IR (KBr) v: $3349(\mathrm{~N}-\mathrm{H}), 1652(\mathrm{C}=$ O) $\mathrm{cm}^{-1}$; MS $\mathrm{m} / \mathrm{z}(\%): 353 \quad\left(\mathrm{M}^{+}, 38\right), 262(3), 91$ (100), 43 (40). Anal. calcd for $\mathrm{C}_{20} \mathrm{H}_{23} \mathrm{~N}_{3} \mathrm{OS}$ : C 67.96, H 6.56, N 11.89; found C 67.82, H 6.51, N 11.97.

3.2.12-烷氧基-3-烷基-5,6,7,8-四氢苯并噻吩并 [2,3- $-d$ 嘧啶-4(3H)酮 $(\mathbf{6 a}, \mathbf{6 c} \sim \mathbf{6 f})$ 的合成

将所得碳二亚胺 $3(3 \mathrm{mmol})$ 加入醇和相应醇的等物 质的量的醇钠溶液 $5 \mathrm{~mL}$, 室温反应 $5 \sim 6 \mathrm{~h}$ ( TLC 监控 反应过程), 反应完成后用乙醇和二氯甲烷 $15 \mathrm{~mL}(V$ : $V=1: 1$ )重结晶, 以较高的产率得到 2-烷氧基-3-烷基$5,6,7,8$-四氢苯并噻吩 $[2,3-d]$ 并嘧啶酮衍生物 $6 \mathbf{a}$ 和 $\mathbf{6 c} \sim$ 6f.

2-乙氧基-3-异丙基-5,6,7,8-四氢苯并噻吩并嘧啶酮 (6a): 白色固体(0.66 g, 76\%). m.p. 89 91 ${ }^{\circ} \mathrm{C} ;{ }^{1} \mathrm{H}$ NMR $\left(\mathrm{CDCl}_{3}, 400 \mathrm{MHz}\right) \delta: 1.36\left(\mathrm{t}, J=7.2,3 \mathrm{H}, \mathrm{CH}_{3}\right) ; 1.48 \sim$ 
$1.52\left(\mathrm{~d}, J=6.8 \mathrm{~Hz}, 6 \mathrm{H}, 2 \times \mathrm{CH}_{3}\right), 1.79 \sim 1.88(\mathrm{~m}, 4 \mathrm{H}, 2 \times$ $\left.\mathrm{CH}_{2}\right), 2.94 \sim 2.68\left(\mathrm{~m}, 4 \mathrm{H}, 2 \times \mathrm{CH}_{2}\right), 4.45 \sim 4.51(\mathrm{~m}, 2 \mathrm{H}$, $\left.\mathrm{OCH}_{2}\right), 5.54$ (bs, $\left.1 \mathrm{H}, \mathrm{NCH}\right) ;{ }^{13} \mathrm{C} \mathrm{NMR}\left(\mathrm{CDCl}_{3}, 100 \mathrm{MHz}\right)$ $\delta: 14.2,20.1,22.4,23.1,25.0,25.6,44.3,64.5,117.6$, $129.3,131.3,153.8,159.2$, 161.7; IR (KBr) $v: 1681(\mathrm{C}=$ O) $\mathrm{cm}^{-1}$; $\mathrm{MS} \mathrm{m} / z$ (\%): $292\left(\mathrm{M}^{+}, 100\right), 249$ (21), 221 (41), 179 (38), 151 (11). Anal. calcd for $\mathrm{C}_{15} \mathrm{H}_{20} \mathrm{~N}_{2} \mathrm{O}_{2} \mathrm{~S}$ : C 61.62, H 6.89, N 9.58; found C 61.54, H 6.77, N 9.44.

2-丁氧基-3-异丙基-5,6,7,8-四氢苯并噻吩并嘧啶酮 (6c): 白色固体(0.68 g, 72\%). m.p. 66 67 ${ }^{\circ} \mathrm{C} ;{ }^{1} \mathrm{H} \mathrm{NMR}$ $\left(\mathrm{CDCl}_{3}, 400 \mathrm{MHz}\right) \delta$ : $0.99\left(\mathrm{t}, J=7.2 \mathrm{~Hz}, 3 \mathrm{H}, \mathrm{CH}_{3}\right), 1.46$ $\left(\mathrm{d}, J=6.8 \mathrm{~Hz}, 6 \mathrm{H}, 2 \times \mathrm{CH}_{3}\right), 1.65 \sim 1.86\left(\mathrm{~m}, 8 \mathrm{H}, 4 \times \mathrm{CH}_{2}\right)$, $2.70 \sim 2.96\left(\mathrm{~m}, 4 \mathrm{H}, 2 \times \mathrm{CH}_{2}\right), 4.42(\mathrm{t}, J=7.2 \mathrm{~Hz}, 2 \mathrm{H}$, $\left.\mathrm{OCH}_{2}\right), 5.54$ (bs, $\left.1 \mathrm{H}, \mathrm{NCH}\right) ;{ }^{13} \mathrm{C} \mathrm{NMR}\left(\mathrm{CDCl}_{3}, 100 \mathrm{MHz}\right)$ $\delta: 13.8,19.4,20.1,22.4,23.2,25.1,30.6,44.3,68.6,117.5$, 129.3, 131.3, 154.0, 159.2, 161.7; IR (KBr) v: $1685(\mathrm{C}=$ O) $\mathrm{cm}^{-1}$; MS m/z (\%): $320\left(\mathrm{M}^{+}, 100\right), 264$ (91), 222 (98), 193 (15), 178 (52), 57 (4). Anal. calcd for $\mathrm{C}_{17} \mathrm{H}_{24} \mathrm{~N}_{2} \mathrm{O}_{2} \mathrm{~S}: \mathrm{C}$ 63.72, H 7.55, N 8.74; found C 63.84, H 7.43, N 8.62.

2-乙氧基-3-丁基-5,6,7,8-四氢苯并噻吩并嘧啶酮 (6d): 白色固体 $(0.74 \mathrm{~g}, 80 \%)$. m.p. $77 \sim 78{ }^{\circ} \mathrm{C} ;{ }^{1} \mathrm{H} \mathrm{NMR}$ $\left(\mathrm{CDCl}_{3}, 400 \mathrm{MHz}\right) \delta: 0.95\left(\mathrm{t}, J=7.2 \mathrm{~Hz}, 3 \mathrm{H}, \mathrm{CH}_{3}\right), 1.35 \sim$ $1.44\left(\mathrm{~m}, 5 \mathrm{H}, \mathrm{CH}_{2} \mathrm{CH}_{3}\right), 1.62 \sim 1.65\left(\mathrm{~m}, 2 \mathrm{H}, \mathrm{CH}_{2}\right), 1.80 \sim$ $1.87\left(\mathrm{~m}, 4 \mathrm{H}, 2 \times \mathrm{CH}_{2}\right), 2.69 \sim 2.98\left(\mathrm{~m}, 4 \mathrm{H}, 2 \times \mathrm{CH}_{2}\right), 4.02$ (t, $\left.J=7.2 \mathrm{~Hz}, 2 \mathrm{H}, \mathrm{NCH}_{2}\right), 4.49$ (t, $\left.J=7.2 \mathrm{~Hz}, 2 \mathrm{H}, \mathrm{OCH}_{2}\right)$; ${ }^{13} \mathrm{C} \mathrm{NMR}\left(\mathrm{CDCl}_{3}, 100 \mathrm{MHz}\right) \delta: 13.8,14.3,20.2,22.4$, $23.2,25.0,25.5,30.4,40.9,64.7,117.4,129.3,131.2$, 153.3, 158.9, 161.9; IR (KBr) v: $1683(\mathrm{C}=\mathrm{O}) \mathrm{cm}^{-1}$; MS $m / z(\%): 306\left(\mathrm{M}^{+}, 100\right), 291$ (10), 278 (7), 250 (24), 222 (18), 206 (20), 178 (23), 151 (16), 57 (36), 43 (76). Anal. calcd for $\mathrm{C}_{16} \mathrm{H}_{22} \mathrm{~N}_{2} \mathrm{O}_{2} \mathrm{~S}$ : C 62.71, H 7.24, N 9.14; found $\mathrm{C}$ 62.43, H 7.13, N 9.02.

2-丙氧基-3-丁基-5,6,7,8-四氢苯并噻吩并嘧啶酮 (6e): 白色固体(0.72 g, 75\%). m.p. $41 \sim 43{ }^{\circ} \mathrm{C} ;{ }^{1} \mathrm{H}$ NMR $\left(\mathrm{CDCl}_{3}, 400 \mathrm{MHz}\right) \delta: 0.95\left(\mathrm{t}, J=7.2 \mathrm{~Hz}, 3 \mathrm{H}, \mathrm{CH}_{3}\right), 1.05(\mathrm{t}$, $\left.J=7.2 \mathrm{~Hz}, 3 \mathrm{H}, \mathrm{CH}_{3}\right), 1.38\left(\mathrm{t}, J=7.2 \mathrm{~Hz}, 2 \mathrm{H}, \mathrm{CH}_{2}\right), 1.62 \sim$ $1.65\left(\mathrm{~m}, 2 \mathrm{H}, \mathrm{CH}_{2}\right), 1.80 \sim 1.87\left(\mathrm{~m}, 6 \mathrm{H}, 3 \times \mathrm{CH}_{2}\right), 2.69 \sim$ $2.98\left(\mathrm{~m}, 4 \mathrm{H}, 2 \times \mathrm{CH}_{2}\right), 4.01 \sim 4.05\left(\mathrm{~m}, 2 \mathrm{H}, \mathrm{NCH}_{2}\right), 4.37(\mathrm{t}$, $\left.J=7.2 \mathrm{~Hz}, 2 \mathrm{H}, \mathrm{OCH}_{2}\right)$; IR $(\mathrm{KBr}) v: 1685(\mathrm{C}=\mathrm{O}) \mathrm{cm}^{-1}$; MS m/z (\%): $320\left(\mathrm{M}^{+}, 95\right), 278$ (75), 261 (66), 222 (100), 206 (56), 194 (40), 179 (97), 151 (85), 57 (18), 43(59). Anal. calcd for $\mathrm{C}_{17} \mathrm{H}_{24} \mathrm{~N}_{2} \mathrm{O}_{2} \mathrm{~S}$ : C 63.72, $\mathrm{H}$ 7.55, N 8.74; found $\mathrm{C} 63.54, \mathrm{H} 7.47, \mathrm{~N} 8.56$.

2-丁氧基-3-丁基-5,6,7,8-四氢苯并噻吩并嘧啶酮 (6f): 白色固体(0.71 g, 70\%). m.p. $61 \sim 62{ }^{\circ} \mathrm{C}$; ${ }^{1} \mathrm{H}$ NMR
$\left(\mathrm{CDCl}_{3}, 400 \mathrm{MHz}\right) \delta: 0.93 \sim 1.00\left(\mathrm{~m}, 6 \mathrm{H}, 2 \times \mathrm{CH}_{3}\right), 1.36 \sim$ $1.41\left(\mathrm{~m}, 2 \mathrm{H}, \mathrm{CH}_{2}\right), 1.46 \sim 1.51(\mathrm{~m}, 2 \mathrm{H}, \mathrm{CH} 2), 1.61 \sim 1.65$ $\left(\mathrm{m}, 2 \mathrm{H}, \mathrm{CH}_{2}\right), 1.76 \sim 1.87\left(\mathrm{~m}, 6 \mathrm{H}, 3 \times \mathrm{CH}_{2}\right), 2.69 \sim 2.98$ $\left(\mathrm{m}, 4 \mathrm{H}, 2 \times \mathrm{CH}_{2}\right), 4.02\left(\mathrm{t}, J=7.2 \mathrm{~Hz}, 2 \mathrm{H}, \mathrm{NCH}_{2}\right), 4.41(\mathrm{t}$, $\left.J=7.2 \mathrm{~Hz}, 2 \mathrm{H}, \mathrm{OCH}_{2}\right)$; IR $(\mathrm{KBr}) v: 1689(\mathrm{C}=\mathrm{O}) \mathrm{cm}^{-1}$; MS m/z (\%): $334\left(\mathrm{M}^{+}, 100\right), 278$ (73), 261 (53), 222 (70), 206 (41), 179 (94), 57 (18), 41 (42). Anal. calcd for $\mathrm{C}_{18} \mathrm{H}_{26} \mathrm{~N}_{2} \mathrm{O}_{2} \mathrm{~S}$ : C 64.64, $\mathrm{H}$ 7.84, N 8.38; found $\mathrm{C} 64.72, \mathrm{H}$ 7.72, N 8.22.

3.2.2 2- 芳氧基-3-烷基-5,6,7,8-四氢苯并噻吩并 $[2,3-d]$ 嘧啶-4(3H)酮 $(\mathbf{7} \sim \mathbf{7} \sim \mathbf{f})$ 的合成

在碳二亚胺 $3(3 \mathrm{mmol})$ 的乙腈溶液中, 加入稍过量 的酚 (3.5 mmol)和无水碳酸钾 $(1.5 \mathrm{mmol})$ 固体, 加热到 $50 \sim 60{ }^{\circ} \mathrm{C}$ 反应 $10 \sim 12 \mathrm{~h}$ (TLC 监控反应过程). 反应完 成后用乙醇和二氯甲烷 $15 \mathrm{~mL}(V: V=1: 1)$ 重结晶进 行纯化, 以 $70 \% \sim 84 \%$ 的产率得到 $7 \mathbf{a} \sim 7 \mathbf{f}$.

2-(2,4-二氯-苯氧基)-3-异丙基-5,6,7,8-四氢苯并噻 吩并嘧啶酩(7a)：白色固体(1.10 g，84\%). m.p. 165 $167{ }^{\circ} \mathrm{C} ;{ }^{1} \mathrm{H}$ NMR $\left(\mathrm{CDCl}_{3}, 400 \mathrm{MHz}\right) \delta$ : 1.61 (d, $J=6.8$ $\left.\mathrm{Hz}, 6 \mathrm{H}, 2 \times \mathrm{CH}_{3}\right), 1.82 \sim 1.83\left(\mathrm{~m}, 4 \mathrm{H}, 2 \times \mathrm{CH}_{2}\right), 2.68 \sim$ $2.98\left(\mathrm{~m}, 4 \mathrm{H}, \quad 2 \times \mathrm{CH}_{2}\right), 5.63(\mathrm{bs}, 1 \mathrm{H}, \mathrm{NCH}), 7.17 \sim 7.51$ (m, 3H, Ar-H); IR (KBr) v: $1687(\mathrm{C}=\mathrm{O}) \mathrm{cm}^{-1}$; MS m/z (\%): 410 (M+1, 46), 408 (100), 366 (56), 331 (82), 323 (15), 303 (19), 288 (85), 205 (13), 43 (26). Anal. calcd for $\mathrm{C}_{19} \mathrm{H}_{18} \mathrm{C}_{12} \mathrm{~N}_{2} \mathrm{O}_{2} \mathrm{~S}$ : C 55.75, H 4.43, N 6.84; found C 55.56, $\mathrm{H} 4.52, \mathrm{~N} 6.72$.

2-(2,6-二氯-4-甲基-苯氧基)-3-异丙基-5,6,7,8-四氢 苯并噻吩并嘧啶酮 (7b): 白色固体(1.00 g, 82\%). m.p. $237 \sim 239{ }^{\circ} \mathrm{C} ;{ }^{1} \mathrm{H}$ NMR $\left(\mathrm{CDCl}_{3}, 400 \mathrm{MHz}\right) \delta: 1.61$ (d, $J=$ $\left.7.2 \mathrm{~Hz}, 6 \mathrm{H}, 2 \times \mathrm{CH}_{3}\right), 1.82 \sim 1.83\left(\mathrm{~m}, 4 \mathrm{H}, 2 \times \mathrm{CH}_{2}\right), 2.21(\mathrm{~s}$, $\left.3 \mathrm{H}, \mathrm{Ar}-\mathrm{CH}_{3}\right), 2.66 \sim 3.00\left(\mathrm{~m}, 4 \mathrm{H}, 2 \times \mathrm{CH}_{2}\right), 5.67(\mathrm{bs}, 1 \mathrm{H}$, $\mathrm{NCH}), 7.19 \sim 7.34(\mathrm{~m}, 2 \mathrm{H}, \mathrm{Ar}-\mathrm{H})$; IR $(\mathrm{KBr}) v: 1680(\mathrm{C}=$ O) $\mathrm{cm}^{-1}$; MS m/z (\%): $425(\mathrm{M}+2,46), 422$ (79), 379 (21), 345 (79), 317 (14), 302 (100), 258 (14), 222 (7), 43 (22). Anal. calcd for $\mathrm{C}_{20} \mathrm{H}_{20} \mathrm{Cl}_{2} \mathrm{~N}_{2} \mathrm{O}_{2} \mathrm{~S}$ : C 56.74, $\mathrm{H}$ 4.76, N 6.62; found $\mathrm{C} 56.54, \mathrm{H} 4.84, \mathrm{~N} 6.52$.

2-(4-甲基-苯氧基)-3-丁基-5,6,7,8-四氢苯并噻吩并 嘧啶酮(7c): 白色固体(0.88 g, 80\%). m.p. 98 100 ${ }^{\circ} \mathrm{C}$; ${ }^{1} \mathrm{H}$ NMR $\left(\mathrm{CDCl}_{3}, 400 \mathrm{MHz}\right) \delta: 0.97(\mathrm{t}, J=4.8 \mathrm{~Hz}, 3 \mathrm{H}$, $\left.\mathrm{CH}_{3}\right), 1.37 \sim 1.53\left(\mathrm{~m}, 2 \mathrm{H}, \mathrm{CH}_{2}\right), 1.65 \sim 1.87(\mathrm{~m}, 6 \mathrm{H}, 3 \times$ $\left.\mathrm{CH}_{2}\right), 2.39\left(\mathrm{~s}, 3 \mathrm{H}, \mathrm{Ar}-\mathrm{CH}_{3}\right), 2.66 \sim 3.00\left(\mathrm{~m}, 4 \mathrm{H}, 2 \times \mathrm{CH}_{2}\right)$, $4.20\left(\mathrm{t}, J=7.2 \mathrm{~Hz}, 2 \mathrm{H}, \mathrm{NCH}_{2}\right), 7.06 \sim 7.24$ (m, 4H, Ar-H); IR (KBr) $v: 1677(\mathrm{C}=\mathrm{O}) \mathrm{cm}^{-1}$; MS m/z (\%): $368\left(\mathrm{M}^{+}, 82\right)$, 351 (17), 311 (32), 269 (100), 204 (53), 176 (22), 91 (70), 77 (48), 64 (42), 57 (29). Anal. calcd for $\mathrm{C}_{21} \mathrm{H}_{24} \mathrm{~N}_{2} \mathrm{O}_{2} \mathrm{~S}$ : C 
68.45, H 6.56, N 7.60; found C 68.54, H 6.43, N 7.53.

2-(2,4-二氯-苯氧基)-3-丁基-5,6,7,8-四氢苯并噻吩 并嘧啶酩 (7d): 白色固体 $(1.00 \mathrm{~g}, 83 \%)$. m.p. 165 $167{ }^{\circ} \mathrm{C} ;{ }^{1} \mathrm{H}$ NMR $\left(\mathrm{CDCl}_{3}, 400 \mathrm{MHz}\right) \delta: 0.98(\mathrm{t}, J=7.2 \mathrm{~Hz}$, $\left.3 \mathrm{H}, \mathrm{CH}_{3}\right), 1.43 \sim 1.49\left(\mathrm{~m}, 2 \mathrm{H}, \mathrm{CH}_{2}\right), 1.78 \sim 1.83(\mathrm{~m}, 6 \mathrm{H}$, $\left.3 \times \mathrm{CH}_{2}\right), 2.67 \sim 3.00\left(\mathrm{~m}, 4 \mathrm{H}, 2 \times \mathrm{CH}_{2}\right), 4.22(\mathrm{t}, J=7.2 \mathrm{~Hz}$, $\left.2 \mathrm{H}, \mathrm{NCH}_{2}\right), 7.22 \sim 7.55$ (m, 3H, Ar-H); IR (KBr) v: 1689 $(\mathrm{C}=\mathrm{O}) \mathrm{cm}^{-1}$; MS m/z (\%): $422\left(\mathrm{M}^{+}, 100\right), 405$ (30), 387 (15), 365 (18), 331 (58), 288 (75), 261 (32), 205 (19), 57 (6). Anal. calcd for $\mathrm{C}_{20} \mathrm{H}_{20} \mathrm{Cl}_{2} \mathrm{~N}_{2} \mathrm{O}_{2} \mathrm{~S}$ : C 55.75, $\mathrm{H} 4.43, \mathrm{~N}$ 6.84; found C 55.56, H 4.52, N 6.72.

2-(2,6-二氯-4-甲基-苯氧基)-3-丁基-5,6,7,8-四氢苯 并噻吩并嘧啶酮 (7e): 白色固体(1.00 g, 80\%). m.p. $178 \sim 179{ }^{\circ} \mathrm{C}$; ${ }^{1} \mathrm{H}$ NMR $\left(\mathrm{CDCl}_{3}, 400 \mathrm{MHz}\right) \delta: 0.9(\mathrm{t}, J=$ $\left.7.2 \mathrm{~Hz}, 3 \mathrm{H}, \mathrm{CH}_{3}\right), 1.45 \sim 1.49\left(\mathrm{~m}, 2 \mathrm{H}, \mathrm{CH}_{2}\right), 1.76 \sim 1.84$ $\left(\mathrm{m}, 4 \mathrm{H}, 3 \times \mathrm{CH}_{2}\right), 2.21\left(\mathrm{~s}, 3 \mathrm{H}, \mathrm{Ar}-\mathrm{CH}_{3}\right), 2.69 \sim 2.99(\mathrm{~m}$, $\left.4 \mathrm{H}, 2 \times \mathrm{CH}_{2}\right), 4.22\left(\mathrm{t}, J=7.2 \mathrm{~Hz}, 2 \mathrm{H}, \mathrm{NCH}_{2}\right), 7.20 \sim 7.33$ (m, 2H, Ar-H); IR (KBr) v: $1683(\mathrm{C}=\mathrm{O}) \mathrm{cm}^{-1}$; MS m/z (\%): $436\left(\mathrm{M}^{+}, 99\right), 419$ (42), 380 (61), 345 (91), 317 (20), 302 (100), 162 (15), 77 (5), 57 (6) $\mathrm{cm}^{-1}$. Anal. calcd for $\mathrm{C}_{21} \mathrm{H}_{22} \mathrm{Cl}_{2} \mathrm{~N}_{2} \mathrm{O}_{2} \mathrm{~S}$ : C 57.67, H 5.07, N 6.40; found C 57.54, $\mathrm{H} 4.99, \mathrm{~N} 6.46$.

2-(4-氯-苯氧基)-3-丁基-5,6,7,8-四氢苯并噻吩并嘧 啶酮(7f): 白色固体(0.97 g, 86\%). m.p. $127{ }^{\circ} \mathrm{C} ;{ }^{1} \mathrm{H}$ NMR $\left(\mathrm{CDCl}_{3}, 400 \mathrm{MHz}\right) \delta: 0.98\left(\mathrm{t}, J=7.2 \mathrm{~Hz}, 3 \mathrm{H}, \mathrm{CH}_{3}\right), 1.42 \sim$ $1.48\left(\mathrm{~m}, 2 \mathrm{H}, \mathrm{CH}_{2}\right), 1.74 \sim 1.84\left(\mathrm{~m}, 6 \mathrm{H}, 3 \times \mathrm{CH}_{2}\right), 2.69 \sim$ $3.00\left(\mathrm{~m}, 4 \mathrm{H}, 2 \times \mathrm{CH}_{2}\right), 4.19\left(\mathrm{t}, J=7.2 \mathrm{~Hz}, 2 \mathrm{H}, \mathrm{NCH}_{2}\right)$, $7.13 \sim 7.42(\mathrm{~m}, 4 \mathrm{H}, \mathrm{Ar}-\mathrm{H})$; IR $(\mathrm{KBr}) v: 1685(\mathrm{C}=\mathrm{O}) \mathrm{cm}^{-1}$; MS m/z (\%): $388\left(\mathrm{M}^{+}, 100\right), 371$ (29), 331 (18), 288 (31), 261 (39), 254 (60), 205 (13), 57 (4). Anal. calcd for $\mathrm{C}_{20} \mathrm{H}_{21} \mathrm{ClN}_{2} \mathrm{O}_{2} \mathrm{~S}$ : C 60.87, H 5.11, N 7.47; found C 60.56, H 5.17, N 7.32.

\section{辅助材料(Supporting Information) 化合物 5, 6, 7 的核}

磁共振氢谱、碳谱. 这些材料可以免费从本刊网站 (http://sioc-journal.cn/)上下载.

\section{References}

[1] (a) Walter, H. WO 99/14202, 1999 [Chem. Abstr. 1999, 130, 252368k].

(b) Walter, H. WO 0034286, 2000 [Chem. Abstr. 2000, 133, 30738d].

[2] (a) Demirayak, S.; Mohsen, U. A. Heterocycl. Chem. 2001, 38, 507. (b) Santagati, A.; Santagati, M.; Modica, M. Heterocycles 1993, 36, 1315.

[3] Jennings, L. D.; Kincaid, S. L.; Wang, Y. D.; Krishnamurthy, G.; Beyer, C. F.; McGinnis, J. P.; Miranda, M.; Discafani, C. M.; Rabindran, S. K. Bioorg. Med. Chem. Lett. 2005, 15, 4731.

[4] Duval, E.; Case, A.; Stein, R. L.; Cuny, G. D. Bioorg. Med. Chem. Lett. 2005, 15, 1885.

[5] Perspicace, E.; Jouan-Hureau, V.; Ragno, R.; Ballante, F.; Sartini, S.; La Motta, C.; Da Settimo, F.; Chen, B.; Kirsch, G.; Schneider, S.; Faivr, B.; Hesse, S. Eur. J. Med. Chem. 2013, 63, 765.

[6] Wu, C.-H.; Coumar, M. S,; Chu, C.-Y.; Lin, W.-H.; Chen, Y.-R.; Chen, C.-T.; Shiao, H.-Y.; Rafi, S.; Wang, S.-Y.; Hsu, H.; Chen, C.-H.; Chang, C.-Y.; Chang, T.-Y.; Lien, T.-W.; Fang, M.-Y.; Yeh, T.-K.; Hsieh, S.-H.; Hsu, J. T.-A.; Liao, C.-C.; Chao, Y.-S.; Hsieh, H.-P. J. Med. Chem. 2010, 53, 7316.

[7] Zeng, G.; Zheng, P. Acta Chim. Sinica 2012. 70(6), 759.

[8] Hu, Y.-G.; Wang, Y.; Du, S.-M.; Chen, X.-B.; Ding, M.-W. Bioorg. Med. Chem. Lett. 2010, 20, 6188.

[9] Hu, Y. G.; Lu, M. Y.; Song, H. L.; Ding, M. W. Chin. J. Org. Chem. 2005, 25, 295 (in Chinese).

(胡扬根, 吕茂云, 宋鹤丽, 丁明武, 有机化学, 2005, 25, 295.)

[10] Hu, Y. G.; Gao, H.-T.; Wang, G.; Wang, Y.; Qu, Y. N.; Xu, J. Chin. J. Org. Chem. 2012, 32, 1468 (in Chinese).

(胡扬根, 高海涛, 王刚, 王燕, 屈永年, 徐靖, 有机化学, 2012, 32,1468 .)

[11] Hu, Y.-G.; Xu, J.; Chen, X.-B.; Qu, Y.-N. Chin. J. Org. Chem. 2009, 29, 1853 (in Chinese).

(胡扬根, 徐靖, 陈小保, 屈永年, 有机化学, 2009, 29, 1853.)

[12] Ding, M.-W.; Yang, S.-J.; Zhu, J. Synthesis 2004, 75.

[13] (a) Zeng, X.-H.; Wang, H.-M.; Cui, Z.-P.; Ding, M.-W.; He, H.-W. Acta Crystallogr. 2006, E62, o228.

(b) Zeng, X.-H.; Yuan, J.-Z.; Huang, N.-Y.; Ding, M.-W.; He, H.-W. Acta Crystallogr. 2005. E61, o4336.

[14] Ding, M. W.; Huang, N. Y.; He, H. W. Synthesis 2005, 1601. 